\title{
Correction to: Schisandrin B Attenuates Inflammation in LPS-Induced Sepsis through miR-17-5p Downregulating TLR4
}

\author{
Zhi-Rong Ji, ${ }^{1}$ Wei-Liang Xue, ${ }^{2,3}$ and Ling Zhang ${ }^{2}$
}

\section{Correction to: Inflammation.}

https://doi.org/10.1007/s10753-018-0931-3

The original version of this article contained mistakes, and the authors would like to correct them. The correct details are given below:

In the first part of the Results section, the subheading "Sch $B$ Increased miR-17-5p and Inflammation and Decreased TLR4 in Sepsis Mice" should be "Sch B Increased miR-17-5p, and Decreased TLR4 and Inflammation in Sepsis Mice".

Publisher's Note Springer Nature remains neutral with regard to jurisdictional claims in published maps and institutional affiliations.

The online version of the original article can be found at https:/doi.org/ 10.1007/s10753-018-0931-3

${ }^{1}$ Department of Traditional Chinese Medicine, People's Hospital of Ningxia Hui Autonomous Region, the First Affiliated Hospital of Northwest University for Nationalities, Yinchuan, 750000, Ningxia, China

${ }^{2}$ Department of Emergency, People's Hospital of Ningxia Hui Autonomous Region, the First Affiliated Hospital of Northwest University for Nationalities, No.301 Zhengyuan North Street, Yinchuan, 750000, Ningxia, China

${ }^{3}$ To whom correspondence should be addressed at Department of Emergency, People's Hospital of Ningxia Hui Autonomous Region, the First Affiliated Hospital of Northwest University for Nationalities, No.301 Zhengyuan North Street, Yinchuan, 750000, Ningxia, China. E-mail: tcmxuejixuan@163.com 